Original Research Paper

\title{
A Guaranteed Time and Rate Control Method for Improving QoS in WLAN
}

\author{
Anitha, A. and J. Jayakumari \\ ${ }^{1}$ Department of Computer Science and Engineering, \\ ${ }^{2}$ Department of Electronics and Communication Engineering, \\ Noorul Islam Centre for Higher Education, Noorul Islam University, Kumaracoil, Tamilnadu, India
}

Article history

Received: 16-01-2016

Revised: 04-03-2016

Accepted: 09-03-2016

Corresponding Author: A. Anitha

Department of Computer

Science and Engineering,

Noorul Islam Centre for Higher

Education, Noorul Islam

University, Kumaracoil,

Tamilnadu, India

Email: anidathi@yahoo.co.in

\begin{abstract}
Wireless Local Area Network is a mobile network, which connects the users in a small specific area. The devices within the area connect with each other through the wireless access points. The main requirements for the connection between the wireless devices are the time and the power. The transmission time between the wireless devices should be less and the power should be effective for the transmission to avoid the loss. With these considerations, we propose a novel method which hybrids the guaranteed time and power. The proposed method is designed for the network, which quickly responds to the slight changes and the normal networks. Guaranteed time slots are used for the time management and the rate control is used for the guaranteed power. This method works well in both the sensitive and insensitive areas. The performance of the proposed work is accurately analyzed using the analytical and the simulation methods. The numerical results obtained is accurate with the simulation results obtained using the ns-2.34 simulator. The throughput obtained is higher for the sensitive areas with minimum delay than the insensitive areas and closely matches the analytical results.
\end{abstract}

Keywords: BER, CAP, CFS, Rate Control

\section{Introduction}

Wireless is the modern alternative, challenges the long established wired networks which are connected by cables. The fundamental wireless network is composed of a number of wireless devices which communicates with each other through the radio frequency waves with 2.4 or $5 \mathrm{GHz}$ band. Wireless Local Area Network (WLAN) is a mobile network, which connects the users in a small specific area. The devices within the area connect with each other through the wireless access points. The WLAN is based on the IEEE 802.11 standard. The standards are compatible, provides interoperability between the devices. IEEE 802.11 is the wireless communication standard used between the wireless stations. The IEEE 802.11 standard versions are $\mathrm{b} / \mathrm{a} / \mathrm{g} / \mathrm{e} / \mathrm{n}$ etc. The IEEE $802.11 \mathrm{~b}$ is the first developed standard, which supports a maximum of $11 \mathrm{Mbps}$ data rate, $2.4 \mathrm{GHz}$ operating frequency and $6.5 \mathrm{Mbps}$ throughput. The IEEE $802.11 \mathrm{n}$ which supports a maximum of $248 \mathrm{Mbps}$ data rate, $2.4 \mathrm{GHz}$ and $5 \mathrm{GHz}$ operating frequency and the throughput depends upon the various factors such as the environment, the channels utilized (20 or $40 \mathrm{MHz}$ ) and the security used.

QoS is defined as the measure of performance for a transmission system that reflects its transmission quality and service availability. QoS in data communication is the power of the network to yield differentiated service to the selected network traffic over various technologies. Nowadays the WLANs are used to deliver high-bandwidth data and time-sensitive multimedia applications which require the need of QoS in WLANs. The QoS parameters for the WLAN are throughput, delay and packet loss. Throughput is the ratio of the packets received to the packets transmitted in Mbps. Delay is the measure of the excess time taken by a packet to reach its destination in ms. Packet Loss $\left(P_{L}\right)$ is defined as:

$$
P_{L}=1-\text { Number of packets transmitted successfully }
$$

Throughput is the required parameter in WLAN due to the overhead within the 802.11 protocol. The overhead is due to the acknowledgements, Contention 
Window and the Interframing parameters. The intense degree of overhead increases due to the increase in PHY data. This requires the improvement of throughput in WLAN. To improve the throughput the rate adaptation mechanism is used in IEEE $802.11 \mathrm{n}$ standard. The IEEE 802.11n is the High Throughput standard with MAC and PHY layer enhancements. The PHY layer is added with Multi Input Multi Output (MIMO) technology with 20 and $40 \mathrm{MHz}$ channels. The MAC layer is enhanced with frame aggregation and block acknowledgements. The rate adaptation is the optimal method to improve the throughput in WLAN. Rate adaptation is the link layer mechanism used to handle/control the multiple transmission rates in the WLAN standard. The 802.11 WLAN standards do not have any inbuilt algorithm to control the multiple transmission rates. Many existing rate adaptation schemes (Biaz and Wu, 2008; Chen et al., 2012; 2007; Holland et al., 2001; Kamerman and Monteban, 1997; Pang et al., 2005; Schmidt et al., 2012; Senthil Kumar and Krishnan, 2010; Wong et al., 2006) has proved their throughput increase using various rate control mechanisms and IEEE standards. Based on the survey of the rate control mechanisms, the primary aim of the authors was in improving the throughput by selecting an optimal rate than in minimizing the delay. As the rate control mechanisms are based on the data rate the priority is given for the throughput improvement than the delay. Various methods (Shen et al., 2014; Senthil Kumar and Krishnan, 2010; Holland et al., 2001) exchanges RTS/CTS control frames, which reduce the collision but increases the delay. The delay sensitive approaches minimize the delay, but increase the retransmissions which in turn increase the communication overhead. To minimize the delay the methods do not use the feedback method for the authentication. The communication is fast with a low delay, but lacks in authentication.

In data communication the layers handshakes with each other for the synchronization between the source and the destination for the error free transmission. These handshakes requires time to propagate across the link, adds latency. The power control is the essential factor in the WLAN to reduce interference in the shared medium, thereby allowing greater re-use and concurrency of communication. The data transfer rate is the base for the power control in WLAN (Shrivastava et al., 2007). The data transfer rate is interrelated with the power, because the size of the data rate and packet size reflects the power utilization in the WLAN networks. The rate adaptation schemes are broadly classified into two types. Open loop type and Closed loop type. The open loop type, the transmitter observes the receiver's channel status and estimates the channel status without expecting any feedback from the receiver. This is the local channel estimation. The data are transmitted based on the success/failure statistics of a particular bit rate, selects the optimum rate and sends the data. The closed loop type, the transmitter receives the feedback information from the receiver using control messages. The channel quality status is given as the feedback to the sender and the sender estimates the bit rates based on the feedback and select the optimum rate, send the packet. The channel quality is estimated based on the drop of packets or by measuring SNR or RSSI. The wireless transmissions by limiting the transmit power of control and data packets, provides opportunity for multiple flows.

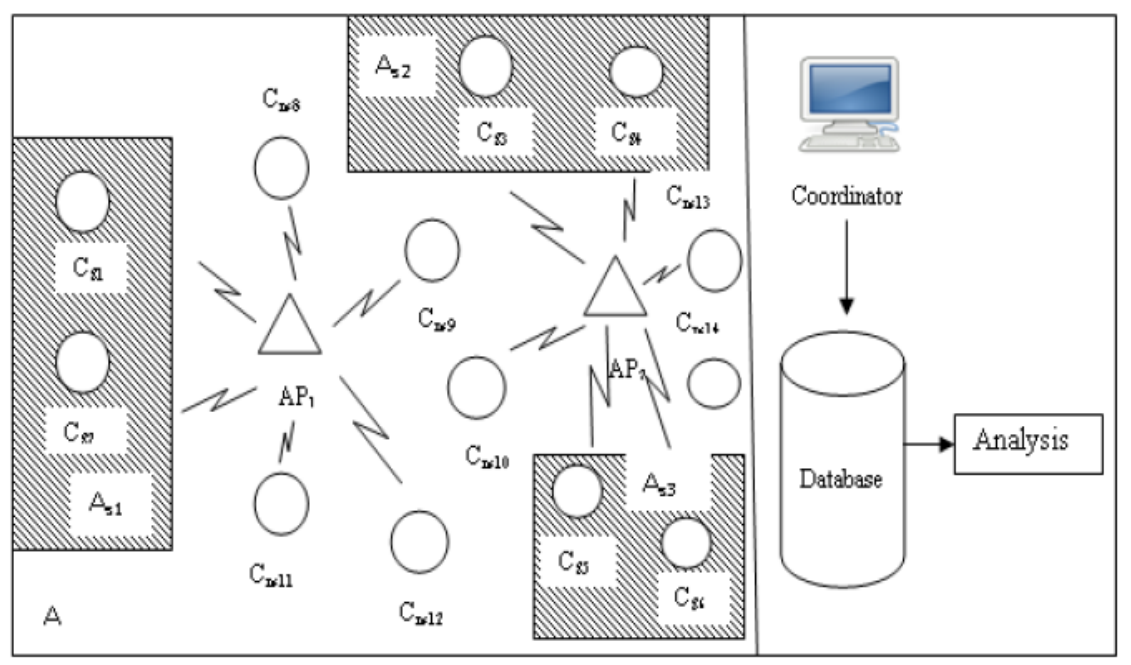

Sensitive Area

Insensitive Area

Fig. 1. Proposed network architecture 
There are multiple distinct approaches for the time control and power control schemes. Both of the approaches have their own merits and demerits. To improve the performance of the WLAN considering the sensitive and insensitive data, a novel method is proposed which hybrids the time control as well as the power control approaches. The proposed method uses the guaranteed time and power which increases the throughput and minimizes the delay. The proposed work mainly aimed for the areas with sensitive data as well as insensitive data. The sensitive data areas, the minute change of the data should be delivered to the destination without a fraction of delay. i.e., the time should guarantee the transmission without delay. The time in WLAN is interrelated with the power of a successful, efficient transmission of the negligible data. For an effective power control the rate control is used to increase/decrease the rate according to the channel condition in the receiver. This is a combined work which combines both the power and time to a guaranteed power and time to achieve best result without the delay and drop. This work can be mainly used in the chemical industries which have the sensitive areas as well as insensitive areas. The proposed work works best for both the sensitive and insensitive areas. The main contribution of our work is based on the sensitive area, uses the guaranteed time and power control algorithms. Figure 1 portrait the proposed network architecture. $A P_{k}$ is the Access Points, $C_{s i}$ are the sensors in the sensitive areas and $C_{n s i}$ are the sensors in the non sensitive areas.

\section{Previous Research}

Makhlouf and Hamdi (2013) proposed a Long Term Stability and Short term responsiveness (L3S) algorithm, an open loop, transmitter based approach. This method used a dynamic probing which adapts to the varying link conditions smartly. It optimizes either the throughput or the delay performance. This method adapts the rate dynamically by predicting the rate based on the history of the statistics. When utilizing both the long-term and the short-term statistics with a small delay the transmitter predicts and tunes to the channel conditions. Ahn and Chung (2015) proposed a Hidden node Effect aware Rate Adaptation (HERA) algorithm, enhanced the performance of WLAN using two factors, namely RTS transmission success/failure and Frame Error Rate (FER). This work analyses the reason for the performance degradation in the hidden node scenario and also addresses the effect of the hidden APs. This work obtained a decrease in aggregated TCP throughput with an increase in the number of hidden nodes.

Shen et al. (2014) proposed a Turborate for the Multiuser MIMO networks. The client in the network has to learn its SNR when it transmits alone to the access point and the direction along which its signal is received at the AP without exchanging the control frames with the AP. Using reciprocity, the clients in the network exploit the beacons to learn the channels from the AP and estimate the reverse channels. To compute the best rate, a Turbo Rate client has to consider the SNR reduction after projecting along the direction orthogonal to all the ongoing transmissions. The SNR reduction after projection depends on the angle between its signal and all the ongoing transmissions.

Lee et al. (2014) proposed a rate adaptation to improve the QoS in VoIP traffic. Vitturi et al. (2013) proposed a rate adaptation scheme for industrial applications decreases the rate for the retransmission, which results in a high degree of frame loss and increases the timing of the successful transmission. Thotahewa et al. (2014), a modified MAC protocol for the wireless sensor networks is proposed. This gives a timeline guarantee to the network. A beacon enabled frame structure used and the timing depends on the data rate and the priority requirements to achieve low power consumption.

\section{Proposed Work}

\section{System Setup}

The proposed work deploys a WLAN with the total area denoted as A. A is divided into the sensitive area $A_{S i}, A_{S i} \in A, i>0$ and the non sensitive area $A_{n s}$. Let $\left\{S_{1}\right.$, $\left.S_{2}, \ldots, S_{n}\right\} \in$ WLAN. A deploys two types of sensor classes in the WLAN. $C_{s}$ is the sensitive class and $C_{n s}$ is the non sensitive class where $C_{s}=\left\{S_{i}, S_{i+1}, \ldots, S_{m}\right\}$, $0<m<n, C_{s} \in A_{S i}$ and $C_{n s}=\left\{S_{m+1}, \ldots \ldots, S_{n}\right\}$. Let $\left\{A P_{1}, \ldots \ldots, A P_{k}\right\}, k>0$ is the Access Points in the WLAN. $A P_{1}=\left\{S_{i}\right\} \subset C_{s}, 1 \leq i \leq m$ and $\left\{S_{j}\right\} \subset C_{n s}, m<j \leq n . A P_{k}=\{$ $\left.S_{i}\right\} \cup\left\{S_{j}\right\}$. The $A P_{k}$ collects data to the database and is analyzed for the various QoS parameters.

\section{Time Slicing}

Figure 2 shows the time slice sequence of the proposed work where $t$ is the time and $\Delta t$ is the time interval. CAP is the Contention Access Period, CFP is the contention Free Period. The channel Access Time of the proposed work is composed of CAP and CFP beacon intervals. The CAP along with the CFP is known as the active period. The AP is active during the whole active period and the nodes in the network is active in the Guaranteed Time Slots (GTS) allocated to them. Maximum 16 time slots are allotted for the active period. The beacon frame occupies the first slot, followed by the CAP slots with a maximum of 7 GTS slots. The source node after the initialization listen the CAP by creating the $R T S_{t p}$. 
During CAP the source node uses the CSMA-CA algorithm to gain the channel access by sending a request using $R T S_{t t}$, time and power guaranteed request to send frame. If the channel access is allocated for the request, the AP indicates the decision to the source node through the next beacon frame by guaranteeing a contention free access. The AP answers the request in 2 steps. First the $\mathrm{AP}$ sends an immediate acknowledgement packet for successful reception of the request to the node without any result of the request. After receiving the acknowledgement the node follows the AP for a specified time. If the AP has enough resources to allocate the GTS to the node, it indicates the node by the next beacon frame with a GTS descriptor containing the address of the requested node, the number and position of the time slots. If the AP has insufficient resources it generates a zero time slot GTS descriptor. If the node does not receive any GTS descriptor it decides the request has failed. Figure 3 shows the elements of the sensitive area and Fig. 4 shows the elements of the insensitive area.

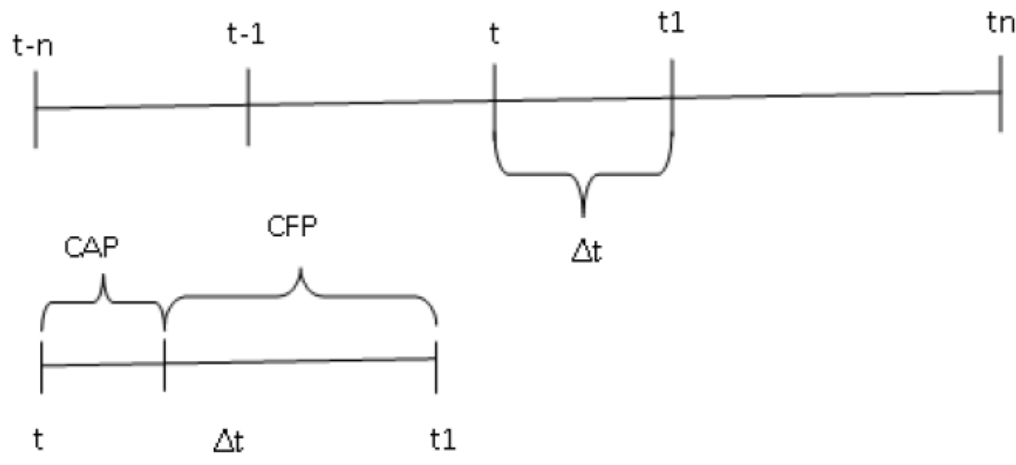

Fig. 2. Time slice sequence

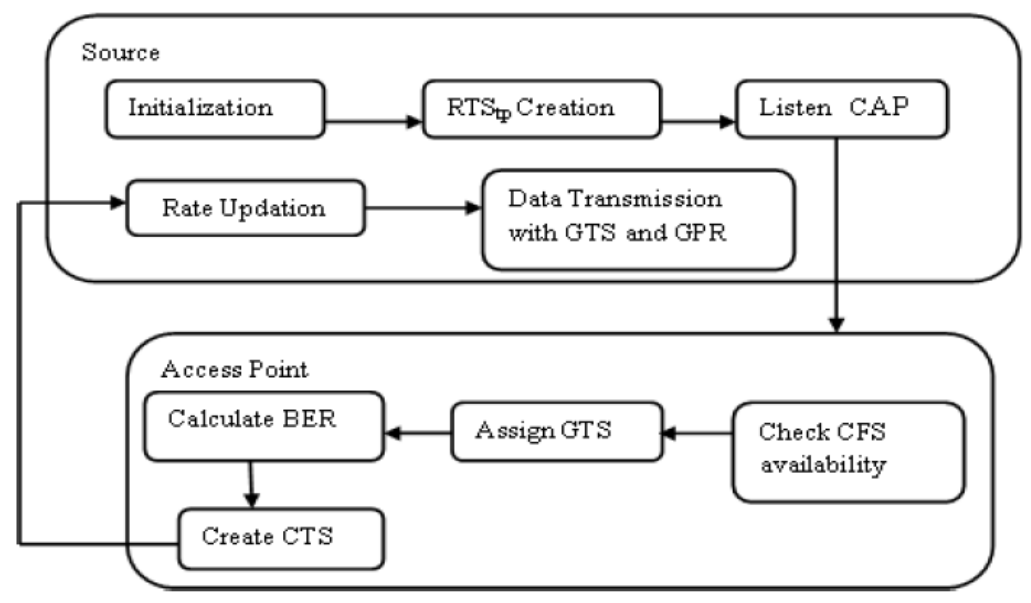

Fig. 3. Elements of the sensitive area algorithm

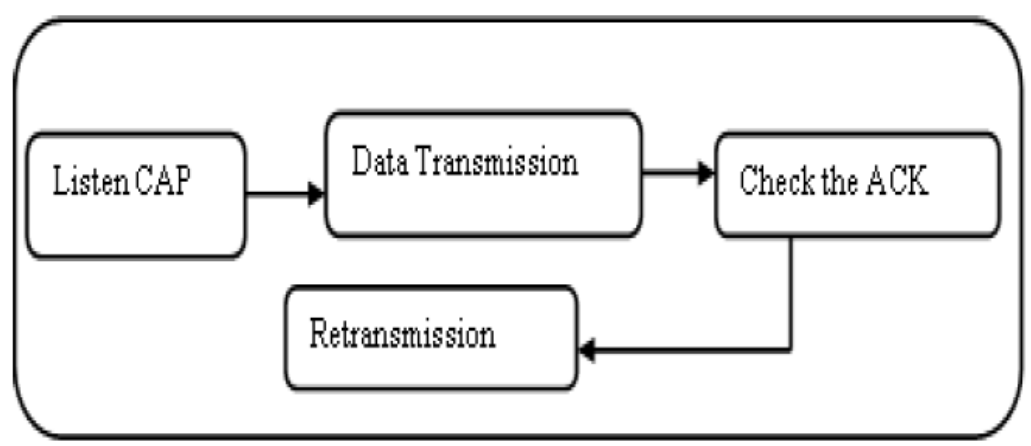

Fig. 4. Elements in insensitive area algorithm 


\section{Initialization}

During the initialization of the sensitive nodes, the source node listen the CAP by requesting the $R T S_{t p}$. If there are sufficient resources, the AP checks the availability of the CFP. If available, the AP assigns GTS, else the source node again listens for CAP. If CFP is available the AP calculates the Bit Error Rate (BER). BER is the ratio of the percentage error bits to the total number of bits transmitted over a given period of time. The BER is derived using the Signal to Noise ratio as follows:

$\gamma=\frac{E_{b}}{N_{0} B T_{b}}$

Where:

$E_{b}=$ The energy/bit

$N_{0}=$ The noise density in $\mathrm{W} / \mathrm{Hz}$

$T_{b}=$ The transmission duration/bit

$B=$ The channel width which is $20 \mathrm{MHz}$ in $802.11 \mathrm{n}$ OFDM PHY. SNR/bit is given as:

$\gamma_{b}=\frac{E_{b}}{N_{0}}=\gamma \cdot B T_{b}=\gamma \cdot \frac{B}{R}$

where, $R=1 / T_{b}$ is the bit transmission rate in Mbps. If the calculated BER is less than the threshold value then the source increase the power level, $P_{r}$. If the BER is greater than the threshold value then the same $P_{r}$ is retained. After the initialization process the AP creates the Clear to Send (CTS) frame which is the combination of both Guaranteed Time Slot (GTS) and Guaranteed Power (GPR). The insensitive nodes listens the CAP. If there is resources to allocate the channel the time slot is allocated by the AP to the requesting source node. Else the node waits for SIFS time interval and again requests for the slot.

\section{Sensitive Area Algorithm}

1. Initialize the source.

2. Source creates $R T S_{t p}$.

3. Source listens CAP.

4. AP checks the availability of CFP.

5. If CFP available, then the AP assigns GTS

Else again source listens CAP.

6. AP calculates BER.

7. IF BER $<$ Thresh, the source changes the $\mathrm{Pr}$ Else retain the same $\mathrm{Pr}$.

8. AP creates $\mathrm{CTS}=\mathrm{GTS}+\mathrm{GPR}$.

9. Data transmission by source with GTS and GPR.

\section{Insensitive Area Algorithm}

1. Source listens CAP.

2. If free slot, data is transmitted to the AP.

3. Check whether the ACK is received.

4. If ACK received, then next data is transmitted. Else retransmission is done.

\section{Analytical Model}

The WLAN considered is assumed with multiple rates and the guaranteed time slot. The data rates are $L_{1}<L_{2}<\ldots \ldots .<L_{n}$ Mbps. The nodes are consistent with the steady state channel conditions. For each rate the nodes are assumed to have the Bit Error Rate (BER), $\gamma_{B E R}$. i.e., $\gamma_{B E R 1} \leq \gamma_{B E R 2} \leq \ldots \ldots . . \leq \gamma_{B E R}$. The number of nodes in the network is assumed as $S_{i}, i \geq 0$. These $S_{i}$ nodes transmits the control and data frames to the AP denoted as $\vartheta_{k} k>0$ through the $R T S_{t p}, \beta_{i}$. The acknowledgement from $\vartheta_{k}$ is given to the $S_{i}$ using $R_{i}$ The time slots CFP, CAP, GTS and GPR is assumed as $\delta, T, \varnothing_{a i(g t s)}, \Psi_{b j(g p r)}$. The $\gamma_{B E R}$ is calculated and compared with the threshold, $\gamma_{T B E R}$. Let $Z$ denotes the data rate of the transmitted rate 5.5 Mbps and $N$ the incremented rate value $11 \mathrm{Mbps}$. The $\vartheta_{k}$ after assigning the time slot and the new rate, $\Re_{n e w}$ informs $S_{i}$ using a CTS, $\wp_{c t s}$. The $S_{i}$ transmits the data, $\rho_{\mathrm{i}}$ to the $\vartheta_{k}$.

The Sensitive algorithm considers $S_{i}$ which adapts to both $A_{s i}$ and $A_{n s}$. Each $S_{i}$ transmits $\beta_{i}, i>0$ to $\vartheta_{k}$, $k>0$. $\vartheta_{k}$ receives $\beta_{i}$ and send $R_{i}$ to $S_{i}, R_{i} \in S_{i}, i>0 \quad \vartheta_{k}$ checks whether $t_{x} \in T, T=\{t-\Delta t\}$ and $\delta \neq \varphi$ then $\vartheta_{k}=$ $\varnothing_{a i(g i s)}, \quad\left\{\varnothing_{a i(g i s)} \neq 0\right\}, \quad 0<\varnothing_{a i(g i s)} \leq 7 . \quad \vartheta_{k}$ calculates $\gamma_{B E R}$ using Equation 2. If $\gamma_{B E R}>\gamma_{T B E R}$ then $\mathfrak{R}_{\text {new }}=N$, else $\Re_{\text {new }}=Z$. $\vartheta_{k}$ creates $\wp_{c t s}=\varnothing_{\text {ai (gts })} \oplus \Psi_{b j(g p r)}, \wp_{c t s} \in \vartheta_{k}$, $S_{i}$ transmits $\rho_{i}, \rho_{i} \in S_{i}$ to $\vartheta_{k}$. If $t_{x} \neq T$, then resend $\beta_{i}$ to $\vartheta_{k}$. The insensitive algorithm in which the $S_{i}$ listens $T$, if $t_{x} \in T, T=\{t-\Delta t\}$ then $S_{i}$ transmits $\rho_{i}$ to $\vartheta_{k}$. If $S_{i}$ receives $R_{i}$, then $S_{i}$ transmits $\rho_{i}+1$ to $\vartheta_{k}$. Else $\rho_{i}$ is retransmitted to $\vartheta_{k}$.

\section{Rate Attempt Model}

The rate control model is assumed as $R_{H R C}$ is a 3tuple $\left(N_{I D},\left\{\varnothing_{a i(g t s)}\right\},\left\{\gamma_{B E T}().\right\}\right)$. Where $N_{I D}=\left\{C_{s i}, 0 \leq i \leq n\right\}$ $=\left\{\varnothing_{a i(g t s)}\right\}=\left\{\varnothing_{a i(g t s)} \neq 0,0<\varnothing_{a i(g t s)} \leq 7\right\},\left\{\gamma_{B E T}().\right\}$ is calculated using Equations 2 and 3. $\vartheta_{k}$ selects the rate for each $C_{s i}$ based on the $R_{i}$ which is dependent on $\left\{\varnothing_{a i(g t s)}\right\}$ for improving the throughput in $A_{s i}$. The rate control can be defined as:

$$
\begin{aligned}
R_{H R C}= & R_{m}, \text { if } \gamma_{B E R}<\gamma T_{B E R} \\
& z R_{n}, \text { if } \gamma_{B E R}>\gamma T_{B E R}
\end{aligned}
$$

$R_{m}$ is the value of the next transmission rate and $\mathrm{z} R_{n}$ is the current transmission rate. 


\section{Throughput Model}

The assumptions for the throughput model are as follows:

- $\quad \mathrm{PA}(\mathrm{t})$ is the packet arrival process and $\lambda$ is the rate of the packets/sec

- Each and every node in the network is with vast buffer space. No drop in packets is caused because of the vast memory storage of the nodes and the packets are transmitted as per the algorithm

- The wireless channels are assumed to be error free. The collision is assumed from the nodes in the $A_{n s}$ area

- The network consists of users with different traffic loads and here two groups are assumed. i.e., $A_{s i}$ and $A_{n s}$

For our throughput model we considered $k$ groups, $k$ $=2$ in the network. Let there are $S_{i}$ nodes in the $k$ group with $\lambda_{i}$ arrival rate. The total number of nodes in each group is assumed to be $\sum_{i=1}^{k} S_{i}=S . A_{s i}$ and $A_{n s}$ consists of number of nodes which forms an independent poisson source with an aggregate Mean packet arrival rate $\lambda_{i}$ for each group such that, $\sum_{i=1}^{k} \lambda_{i}=\lambda$. The probability of successful transmission $T_{p r}$, for $S$ number of nodes with a probability of $\rho$ is defined as:

$$
T_{p r}=1-(1-\rho)^{s}
$$

With a probability of at least one transmission in a given slot of $T$. A successful packet transmission in a group $k$ should be free from the interferences occurred by the nodes in the $k$ groups and should satisfy the following conditions:

- A packet is transmitted successfully within the $\mathrm{k}$ group with a probability, $P_{k s}(k)$

- A packet is transmitted successfully in all groups, $m$ with a probability, $P_{k s}(m), m \neq k$

A packet transmission is successful only when a node in the single group attempts to transmit based on the channel condition and the $\gamma_{B E R}$ calculated at $\vartheta_{k}$. The probability of success is defined by:

$$
P_{k s}=k \rho(1-\rho)^{k-1} / T_{p r}
$$

The normalized system throughput:

$$
\begin{aligned}
& S T=\frac{E[\text { payload in formationin the slot time }]}{E[\text { Lengthof the slot time }]} \\
& S T=\frac{P_{k s} T_{p r} E[p]}{\left(1-T_{p r}\right) \phi+P_{k s} T_{p r} T_{s}+\left(1-p_{k s}\right) T_{p r} T_{c}}
\end{aligned}
$$

Here $E[P]$ is the average packet payload size. $\mathrm{P}_{\mathrm{ks}} T_{p r}$ $E[P]$ is the payload time which successfully transmits the average payload information with the probability $\mathrm{P}_{\mathrm{ks}} T_{p r}$. $\varnothing$ is the empty slot time duration. $T_{s}$ is the average time the channel is busy for a successful transmission by a node. $T_{c}$ is the average time the channel is busy for the nodes during collision.

The values of $T_{s}$ and $T_{c}$ varies for the $A_{s i}$ and $A_{n s}$. The $A_{n s}$ access uses the basic access mechanism and the $T_{s}$ is denoted as $T_{s}^{\text {insen }}, T_{c}$ is denoted as $T_{c}^{\text {insen }}$, the values are referred from (Bianchi, 2000) as follows:

$$
\begin{aligned}
T_{s}^{\text {insen }} & =H+E[p]+S I F S+\partial+A C K+D I F S+\partial \\
& T_{c}^{i n s e n}=H+E[P *]+D I F S+\partial
\end{aligned}
$$

The packet header, $H=P H Y_{h d r}+M A C_{h d r}$ and $\partial$ is the propagation delay. The $A_{s i}$ uses the RTS/CTS mechanism for the $\varnothing_{a i(g t s)} \oplus \Psi_{b j(g p r)}$ allotment in the $\delta$ interval. The $T_{s}$ is denoted as $T_{s}^{\text {sen }}$ and the $T_{c}$ is denoted as $T_{c}^{\text {sen }}$ :

$$
\begin{aligned}
& T_{s}^{s e n}=R T S+S I F S+\partial+A C K+D I F S+G T S+\partial+C T S \\
& +S I F S+\partial+H+E[p] \\
& T_{c}^{s e n}=R T S+D I F S+\partial
\end{aligned}
$$

GTS is the time duration to allot the $\varnothing_{a i(g t s)}, \Psi_{b j(g p r)}$ and $\gamma_{B E R}$ calculation. The collision is assumed to occur only during the RTS transmission.

\section{Delay Analysis}

The delay model considered the IEEE WLAN which consists of $\mathrm{n}$ nodes which contend under ideal channel condition. Each station is assumed with a packet for transmission its transmission queue. The probability of, $p$ is constant and independent of the number of collisions that the packet has suffered.

The average delay $E[D]$ is given by:

$$
E[D]=E[S] . E[\text { length of a slot time }]
$$

where, $E[S]$ is the average number of slot times required for the successful transmission of a new frame. E[lengts of a slot time $]$ is same as Equation $8 . E[S]$ is given as:

$$
E[S]=\frac{(1-2 p) \cdot(w+1)+p w \cdot\left(1-(2 p)^{m}\right)}{2 \cdot(1-2 p) \cdot(1-p)}
$$

\section{Performance Evaluation}

This paper mainly concentrates on the throughput and the delay performance of the WLAN networks which considers both the sensitive and insensitive areas. The 
sensitive areas should be able to transmit its data to the destination with minimum delay. For the efficient transmission of the sensitive data to the destination the guaranteed time slot and power is allotted using the proposed algorithm. The insensitive area nodes access is same as the basic access method. The analytical model of the proposed work is modeled and this result is compared with the simulation results. The parameters used by the proposed model are given in Table 1 . The infrastructure based WLAN 802.11n is considered. The performance analysis is done by varying the number of nodes with the fixed rate $5.5 \mathrm{Mbps}$. The throughput and delay is obtained separately for the sensitive area nodes and the insensitive area nodes.

Figure 5 portraits the throughput performance of the insensitive area in which the process is similar to the basic access method. Here the RTS/CTS is exchanged between the AP and the source node and the data is transmitted. If the acknowledgement is received then the next data is transmitted else the previous data is retransmitted. The throughput obtained for the analysis model closely matches with the simulation model with a difference of \pm 0.002 . The insensitive area nodes due to the collision, more retransmission of the data and more waiting time the throughput obtained is not up to the mark. The throughput decreases with the increase in number of nodes. This shows that the performance of the insensitive nodes in the network depends on the size of the network. This is due to the size of the Contention Window is acceptable for the small number of nodes and does not match for the higher number of nodes which drastically affects the throughput.
The throughput results for the sensitive area are given in Fig. 6. The analytical results obtained are the accurate numerical results and it closely matches with the simulation results. The throughput obtained is higher for the smaller number of nodes and decreases as the number of nodes increases. But the throughput obtained is higher than the insensitive areas. The sensitive nodes obtain high throughput because of the guaranteed time slot and the rate control in the AP. The collision may occur only during the RTS request and not during the data transmission. The throughput results for the number of nodes in both the sensitive and the insensitive nodes depends on the transmission probability and it varies for each set of anodes as well as the methods and is given in Fig. 7.

Table 1. Parameters to obtain numerical values both for the analytical and simulation models

\begin{tabular}{ll}
\hline Packet payload & $8184 \mathrm{bits}$ \\
\hline MAC header & $271 \mathrm{bits}$ \\
PHY header & $128 \mathrm{bits}$ \\
Acknowledgement & $240 \mathrm{bits}$ \\
RTS & $288 \mathrm{bits}$ \\
CTS & $240 \mathrm{bits}$ \\
Channel bit rate & $1 \mathrm{Mbps}$ \\
Propagation Delay & $1 \mu \mathrm{s}$ \\
Slot Time & $50 \mu \mathrm{s}$ \\
SIFS & $28 \mu \mathrm{s}$ \\
DIFS & $128 \mu \mathrm{s}$ \\
Acknowledgement Time out & $300 \mu \mathrm{s}$ \\
CTS Time out & $300 \mu \mathrm{s}$ \\
GTS & $250 \mu \mathrm{s}$ \\
\hline
\end{tabular}

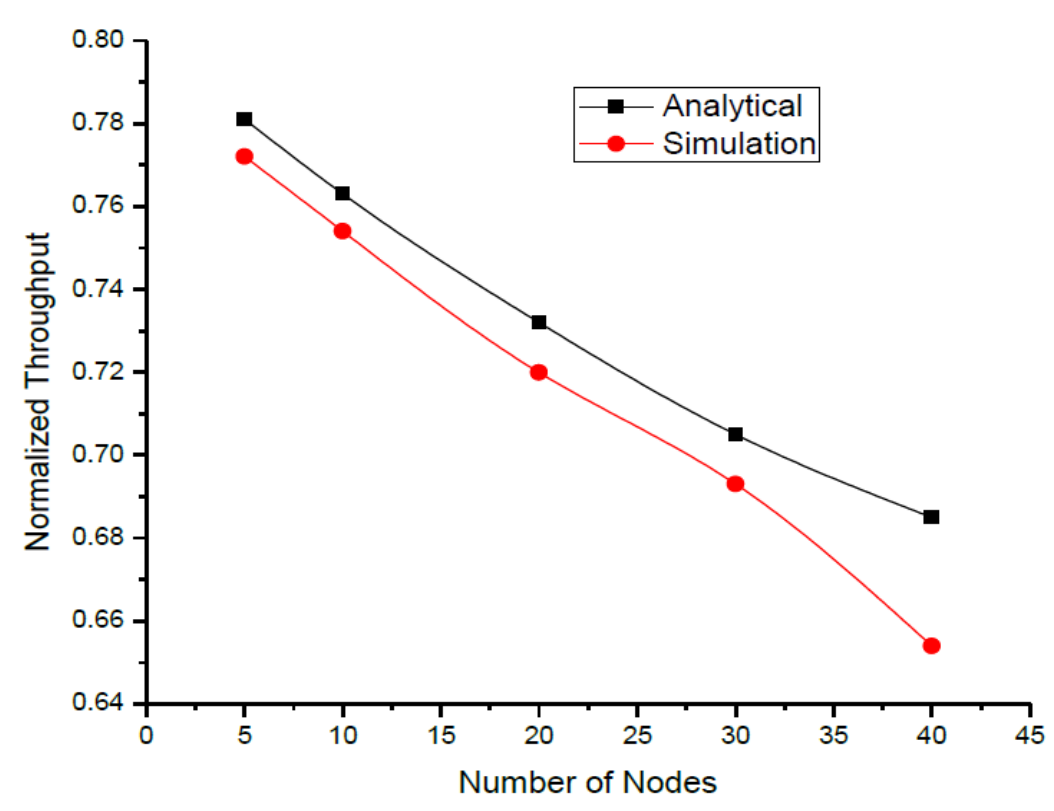

Fig. 5. Throughput results for the insensitive areas with the varying number of nodes 


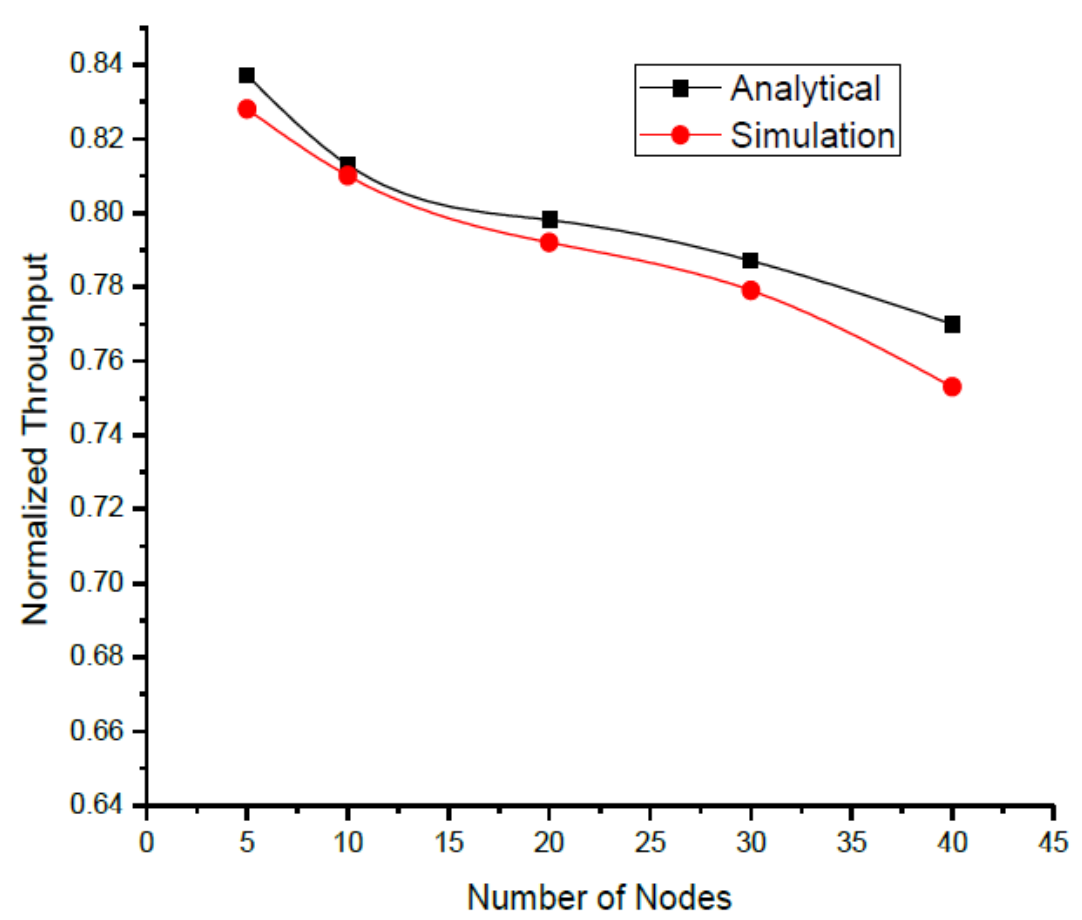

Fig. 6. Throughput results for the sensitive areas with varying number of nodes

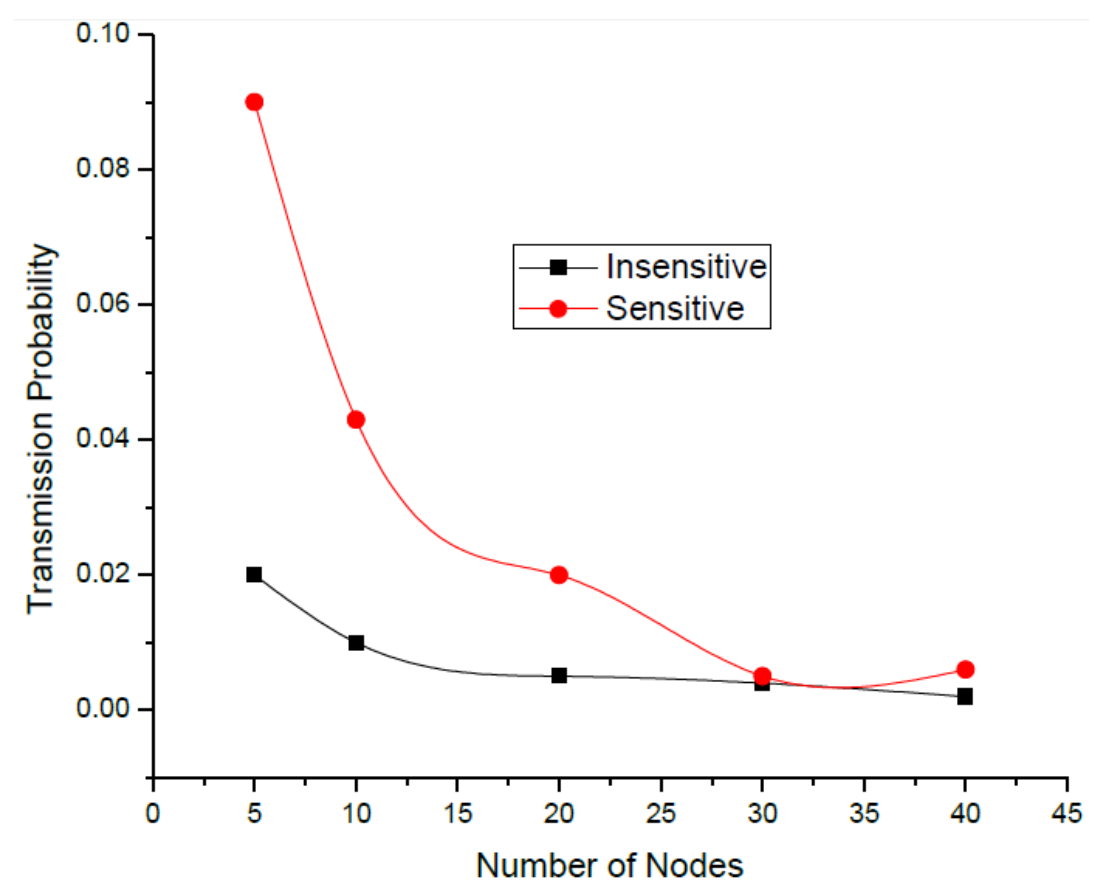

Fig. 7. Transmission probabilities for the various numbers of nodes

The sensitive nodes achieves high transmission probability than the insensitive nodes due to the $P_{k s}$ $T_{p r}$ i.e., for a successful transmission the average number of time slot is given as $1 /\left(P_{k s} T_{p r}\right)$. The average number of idle slot is given by $\left(1-T_{p r}\right) /\left(P_{k s}\right.$
$\left.T_{p r}\right)$. The performance is higher than the existing methods because of the hybrid combination of the time and power guaranteed network which reduces collision and increases the transmission probability for the emergency areas. 


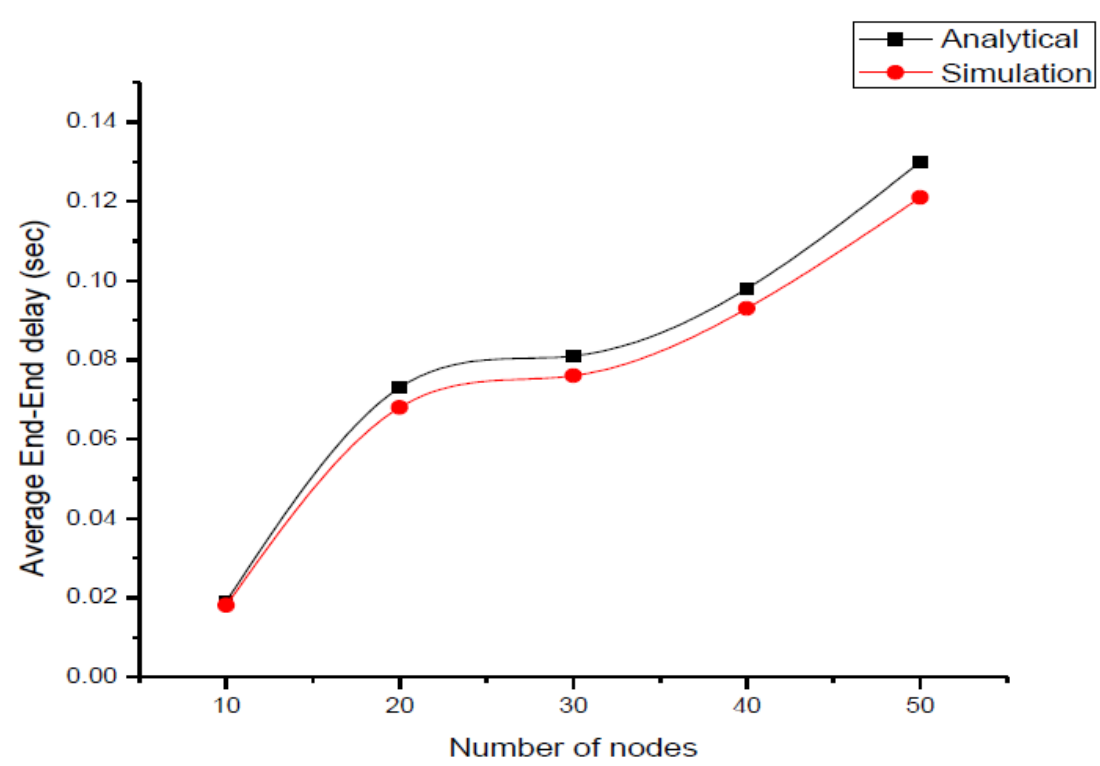

Fig. 8. Delay analysis for the sensitive locations with varying number of nodes

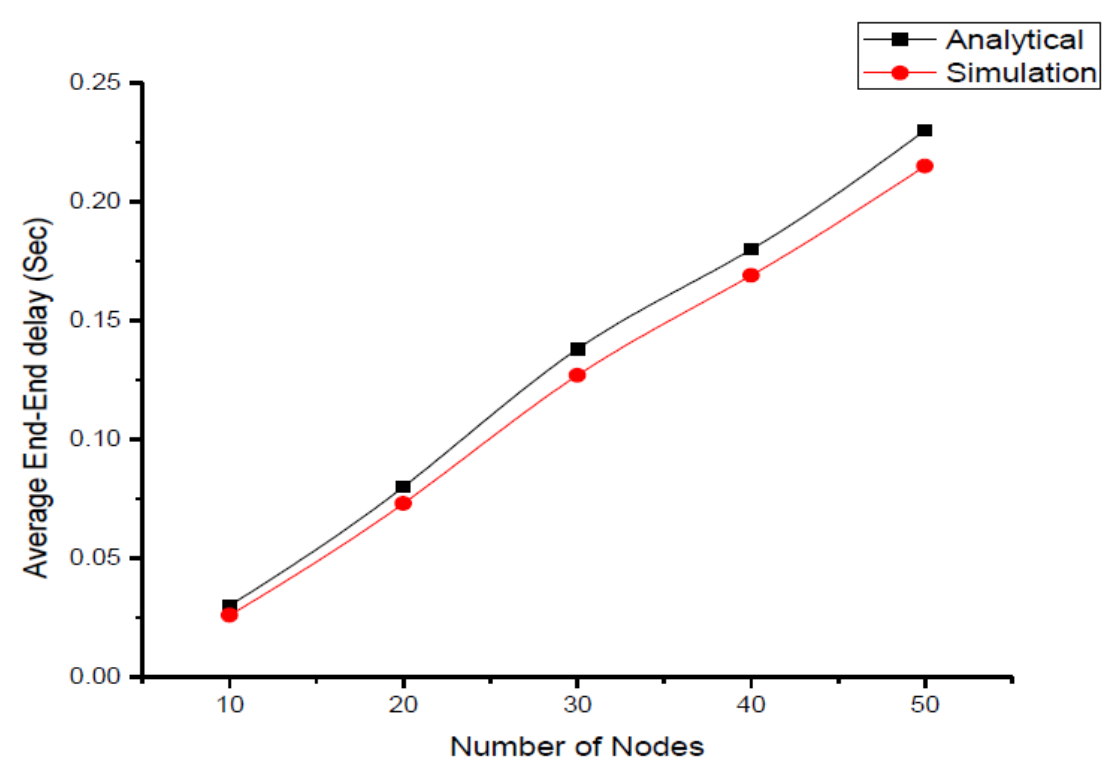

Fig. 9. Delay analysis for the insensitive locations with varying number of nodes

Figure 8 portraits the delay analysis of the sensitive nodes. The results obtained where accurate with the analytical model. The delay increased with the increase in number of nodes. But the delay obtained is very low compared to the existing methods due to the guaranteed time allotted and the rate control by our algorithm which gave the nodes to transmit with the minimum delay. Figure 9 portraits the delay analysis for the insensitive nodes which gave the delay than the sensitive nodes due to the lack of the guaranteed time and the basic access mechanisms and more retransmissions.

\section{Conclusion}

This is the hybrid method of both guaranteed time and power suitable for both the sensitive and insensitive nodes in a WLAN network. This paper presents the analytical and the simulation model for the proposed work. This method proposes the rate control method with the guaranteed time slot to reduce the delay of data transmission and to increase the successful transmission probability. Both the analytical and the simulation model achieved best results and is best than the existing rate control methods. This work 
can be used in the emergency areas where the transmission of success should be increased.

\section{Funding Information}

The authors have no funding to report.

\section{Author's Contributions}

A. Anitha: Performed the simulation, analytical method for the proposed work and contributed to the writing of the manuscript.

J. Jayakumari: Supervisor of the work and reviewed the manuscript.

\section{Ethics}

This manuscript is original and contains unpublished materials. The corresponding author confirms that all of the authors have read and approved the manuscript.

\section{References}

Ahn, C.W. and S.H. Chung, 2015. Enhancing WLAN performance with rate adaptation considering hidden node effect. Int. J. Distrib. Sensor Netw. DOI: $10.1155 / 2015 / 940505$

Bianchi, G., 2000. Performance analysis of the IEEE 802.11 distributed coordination function. IEEE J. Selected Areas Commun., 18: 535-548. DOI: $10.1109 / 49.840210$

Biaz, S. and S. Wu, 2008. ERA: Effective rate adaptation for WLANs. Lecture Notes Comput. Sci., 4982: 901-913. DOI: 10.1007/978-3-540-79549-0_79

Chen, X., D. Qiao, J. Yu and S. Choi, 2007. Probabilistic-based rate adaptation for IEEE 802.11 WLANs. Proceedings of the IEEE Global Telecommunications Conference, Nov. 26-30, IEEE Xpore Press, Washington, DC., pp: 4904-4908. DOI: $10.1109 /$ GLOCOM.2007.930

Chen, X., P. Gangwal and D. Qiao, 2012. RAM: Rate adaptation in mobile environments. IEEE Trans. Mob. Comput., 11: 464-477. DOI: 10.1109/TMC.2011.91

Holland, G., N. Vaidya and P. Bahl, 2001. A rateadaptive MAC protocol for multi-hop wireless networks. Proceedings of the 7th Annual International Conference on Mobile Computing and Networking, Jul. 16-21, Rome, Italy, pp: 236-251. DOI: $10.1145 / 381677.381700$

Kamerman, A and L. Monteban, 1997. WaveLAN®-II: A high-performance wireless LAN for the unlicensed band. Bell Labs Technical J., 2: 118-133. DOI: $10.1002 /$ bltj.2069
Lee, H., S. Byeon, B. Kim, K.B. Lee and S. Choi, 2014. Enhancing voice over WLAN via rate adaptation and retry scheduling. IEEE Trans. Mob. Comput., 13: 2791-2805. DOI: 10.1109/TMC.2013.54

Makhlouf, A.B. and M. Hamdi, 2013. Practical rate adaptation for very high throughput wlans. IEEE Trans. Wireless Commun., 12: 908-916. DOI: 10.1109/TWC.2013.13.120626

Pang, Q., C.M. Leung and C. Liew, 2005. A rate adaptation algorithm for IEEE 802.11 WLANs based on MAC-layer loss differentiation. Proceedings of the 2nd International Conference on Broadband Networks, Oct. 3-7, IEEE Xplore Press, pp: 659-667. DOI: 10.1109/ICBN.2005.1589671

Schmidt, F., A. Hithnawi, O. Punal, J. Gross and K. Wehrle, 2012. A receiver-based 802.11 rate adaptation scheme with on-demand feedback. Proceedings of the 23rd International Symposium on Personal Indoor and Mobile Radio Communications, Sept. 9-12, IEEE Xplore Press, Sydney, NSW, pp: 399-405. DOI: 10.1109/PIMRC.2012.6362818

Senthil Kumar, D. and A. Krishnan, 2010. Throughput analysis of IEEE 802.11 multirate WLANs with collision aware rate adaptation algorithm. Int. J. Automation Comput., 7: 517-577. DOI: $10.1007 / \mathrm{s} 11633-010-0542-4$

Shen, W., K. Lin, S. Gollakota and M. Chen, 2014. Rate adaptation for 802.11 multiuser MIMO networks. IEEE Trans. Mobile Comput., 13: 35-47.

Shrivastava, V., D. Agrawal, A. Mishra and S. Banerjee, 2007. Understanding the limitations of transmit power control for indoor WLANs. Proceedings of the 7th ACM SIGCOMM Conference on Internet Measurement, (CIM' 07), pp: 351-364.

Thotahewa, M.S., Y. Khan and R. Yuce, 2014. Power efficient ultra wide band based wireless body area networks with narrowband feedback path. IEEE Trans. Mob. Comput., 13: 1829-1842.

DOI: $10.1109 /$ TMC.2013.120

Vitturi, S., L. Seno, F. Tramarin and M. Bertocco, 2013. On the rate adaptation techniques of IEEE 802.11 networks for industrial applications. IEEE Trans. Industrial Inform., 9: 198-208. DOI: 10.1109/TII.2012.2189223

Wong, H.Y., H. Yang, S. Lu and V. Bharghavan, 2006. Robust rate adaptation for 802.11 wireless networks. Proceedings of the 12th Annual International Conference on Mobile Computing and Networking, Sept. 24-29, Los Angeles, CA, USA, pp: 146-157. DOI: $10.1145 / 1161089.1161107$ 